Method We studied 61 2-8 year-old children (including 7 with Down's syndrome) attending audiology clinics with OME $(n=38)$ and normal hearing $(n=23)$. Children and parents were observed and questioned during their first use of the app to assess acceptability and usability. One week later, they were contacted about home use. Additionally, 18 clinicians completed questionnaires regarding use of the app in managing OME.

Results $80 \%$ of children liked the app and $90 \%$ found it easy to use. In children with Down's Syndrome, accessibility depended on developmental age and previous app experience rather than chronological age.

$89 \%$ of parents wanted their child to use the app regularly. Despite finding no significant correlation between the app's hearing screen and audiology clinic results $(p=0.381)$, $80 \%$ of parents felt more confident gauging and communicating their child's hearing levels. $89 \%$ of families used the app at home and $75 \%$ felt it helped their child's speech development.

$78 \%$ of clinicians would like patients to use the app regularly, and $89 \%$ believed it would help parents support their child's development.

Conclusions The 'Hear Glue Ear' app is acceptable to children, parents and clinicians. It offers an affordable and accessible means to monitor hearing at home, deliver speech and language therapy remotely, and support speech development during the 'watchful waiting' period of OME management.

\section{G266(P) A REGIONAL QUALITY IMPROVEMENT PROJECT FOR CONGENITAL CYTOMEGALOVIRUS (CCMV) ASSOCIATED SENSORINEURAL HEARING LOSS LEADING TO IMPROVED PRIORITISATION OF FINITE RESOURCES DURING THE COVID-19 PANDEMIC}

${ }^{1} \mathrm{Z}$ Khan, ${ }^{2} \mathrm{R}$ Brown, ${ }^{3} \mathrm{~V}$ Banks, ${ }^{4} \mathrm{~T}$ Holland Brown. ${ }^{1}$ University of Cambridge School of Medicine; ${ }^{2}$ Paediatrics, Addenbrooke's Hospital, Cambridge; ${ }^{3}$ Newborn Hearing Screening, Addenbrooke's Hospital, Cambridge; ${ }^{4}$ Community Paediatrics, Cambridge Community Services NHS Trust, Cambridge, UK

\subsection{6/archdischild-2020-rcpch.230}

Background Congenital CMV [cCMV] is a leading cause of hearing loss and childhood disability. Recent research demonstrated children's long-term outcomes could be improved with antiviral treatment offered within the first month of life. During the COVID-19 pandemic, national hearing screening guidance promoted prioritisation of face-to-face diagnostic auditory brainstem response $[A B R]$ in infants with cCMV.

Aim To identify babies with cCMV and hearing loss within 1 month of life to ensure:

- Greater certainty of the diagnosis of cCMV.

- Early paediatric referral for identification and management of cCMV

- Timely consideration of treatment

Method A truly joined-up, multidisciplinary approach was taken across the region (newborn hearing screeners, audiologists, ENT surgeons, virologists, neonatologist, paediatricians, ophthalmologists). Targeted cCMV testing was undertaken by newborn hearing screeners in all babies who 'failed' their otoacoustic emission and automated ABR. CMV positive babies were referred urgently to paediatricians to consider management and treatment within 1 month of life. We derived data on the timing of cCMV testing in the months before and then during the pandemic.

Results Regional hospitals worked together to write the East of England CMV guidelines which were then ratified. https:// www.networks.nhs.uk/nhs-networks/eoe-neonatal-odn/guidelines/ current-guidelines/congenital-cmv-guideline

Following implementation in early 2019, Addenbrookes Hospital data showed that babies undergoing hearing screening during the pandemic could be prioritised according to national guidance for definitive ABR testing

Conclusion CMV saliva swabs for babies born with hearing loss was implemented across the East of England region using a wide multidisciplinary approach. In the pandemic, this enabled prioritisation of diagnostic ABR testing in babies with $\mathrm{cCMV}$ and hearing impairment according to national guidance.

\section{Down syndrome medical interest group, british association of community child health and british paediatric respiratory society}

\section{G267 PREVENTING INFECTION AND MORTALITY IN DOWN SYNDROME - THE NOTTINGHAM EXPERIENCE}

${ }^{1,2}$ AP Prayle, ${ }^{1} P$ Shires, ${ }^{3} V$ Baicher, ${ }^{1} S$ Smith, ${ }^{3} L$ Walton, ${ }^{4} E$ McDermott, ${ }^{1} E$ Marder. ${ }^{1}$ Family Health, Nottingham University Hospitals NHS Trust, Nottingham, UK; ${ }^{2}$ Child Health, The University of Nottingham, Nottingham, UK; ${ }^{3}$ Emergency Department, Nottingham University Hospitals NHS Trust, Nottingham, UK; ${ }^{4}$ Immunology, Nottingham University Hospitals NHS Trust, Nottingham, UK

\subsection{6/archdischild-2020-rcpch.231}

Purpose Children with Trisomy 21 are at increased risk of death compared to age-matched controls. We recently undertook a retrospective review of mortality in Trisomy 21 in our tertiary non-cardiac centre, and found an infective cause of death in $9 / 16$ cases.

Methods In response to these data, we have undertaken a number of interventions at the Trust level with the goal of reducing the risk of future mortality and morbidity.

- New local guidance for preventing infection in Trisomy 21

- Aggressively treating comorbidities such as reflux and sleep related breathing disorders

- Protocol driven assessment of immune function including functional antibodies and meticulous immunisation

- We recommend influenza and pneumovax II for all

- An alert on the Trust wide computer system for patients when they present in the emergency department or on the Children's Assessment Unit to emphasise the risk of infection in Trisomy 21

- A review of how often and why children with Trisomy 21 present to ED

- Increasing awareness for parents, including an alert in the Down syndrome insert for the parent held child health record (the 'red book')

- A standardized alert sentence on all letters to primary care from the Trisomy 21 clinic emphasizing the risk of infection

- Increasing awareness amongst Clinicians, through programmed teaching, bulletins, and alerts 
Conclusions We have instituted a series of measures to increase awareness of infection and sepsis in Trisomy 21, and to reduce the risk of serious bacterial infection. This is in direct response to local data on the causes of mortality in Trisomy 21 in our centre. Whilst it is too early to assess the results of the implemented measures, our experience shows that a multifaceted, multi-departmental approach to reducing risk of mortality can be implemented.

\section{G268 THE JAMES LIND ALLIANCE AND CYSTIC FIBROSIS: A JOURNEY TOWARDS CO-PRODUCTION OF RESEARCH}

${ }^{1}$ NJ Rowbotham, ${ }^{1}$ S J Smith, ${ }^{2}$ ZC Elliott, ${ }^{1}$ AR Smyth. ${ }^{1}$ Evidence Based Child Health, Div. Child Health, Obstetrics and Gynaecology, University of Nottingham, Nottingham, UK; ${ }^{2}$ Parent of Children with Cystic Fibrosis, Nottingham, UK

10.1136/archdischild-2020-rcpch.232

Cystic Fibrosis (CF) is the commonest life limiting inherited condition in the UK, affecting 3895 children. Children with $\mathrm{CF}$ undertake burdensome, time-consuming treatment, much of which is not evidence-based. Questions for clinical research should reflect patient and clinician priorities because both the number of potential participants and funding are limited.

Aims We describe our work over a 4 year period to engage the CF community in prioritisation and co-production of clinical research.

Methods In 2016-17 we undertook a James Lind Alliance (JLA) Priority Setting Partnership (PSP) to find the top 10 priorities for clinical research in CF. Through a series of surveys and workshops these were agreed in conjunction with the global CF community. In 2018-2019 we undertook further global surveys to explore 4 of the top 10 questions in more depth (treatment burden, gastrointestinal symptoms, adherence and exercise in place of some airway clearance). To avoid cross-infection and increase global reach, we adapted JLA methodology to use video conferencing and social media.

Results During the past four years we have gathered opinions from 3729 respondents across six continents. Our youngest respondents were six years old and the median age of respondents with CF (answering directly or by proxy) was 15 years. On completion we disseminated the top 10 questions widely and worked to raise awareness with research funders such as the NIHR. This has resulted in two NIHR themed calls, featuring many of the JLA CF top 10 questions.

Conclusions Encouraging patient participation, including children, in all stages of evidence-based medicine is vital to ensure clinical research is of good quality and relevant to patient needs.

\section{G269 CHAMPIONING THE EX-PRETERM CHILD AT SCHOOL}

FE Payne, AM Oliver. Community Paediatrics, Barnsley Hospital, Barnsley, UK

10.1136/archdischild-2020-rcpch.233

Aims Children born prematurely are more likely to experience health, educational and social difficulties compared to matched term peers. Educational professionals lack relevant knowledge and feel unprepared to support them.

This project evaluates local Special Educational Needs Coordinators (SENCOs) existing knowledge; develops and delivers a teaching package, and evaluates the effectiveness of this resource.

Method

- Initial survey developed and sent to all local primary school SENCOs.

- Teaching package developed.

- Teaching revised based on SENCOs and educational psychologist focus group feedback.

- Prematurity teaching package delivered to the regional Newly Qualified Teachers training.

- Post-teaching survey distributed and analysed.

Results

- $27 \%(22 / 77)$ of SENCOs completed the initial survey.

- $86 \%$ reported little/no previous training.

- $86 \%$ reported limited/no knowledge of the education and social effects.

- $67 \%$ reported school had no process for routinely identifying ex-preterms.

- Qualitative data, in response to 'improving health and education working':

- $12 / 33$ responses requested 'training about prematurity effects' ( $95 \%$ felt face to face with/without written format would be optimal).

- The final teaching package was delivered to 37 teachers.

- Post-teaching survey showed an improvement in perceived: educational difficulties - 28/37 rated their knowledge after as good/excellent compared to $5 / 37$ before social difficulties $30 / 37$ rating their knowledge after as good/excellent compared to $5 / 37$ before $95 \%$ felt the teaching was

- 'useful'/'very useful'.

- $100 \%$ reported it would change their future approach. The most common thematic responses included 'identifying', 'asking parents' and 'providing support'.

- 95\% reported preference for face-to-face training over electronic. 'Questions', 'explanations' and 'engaging' were the most common qualitative themes.

- Feasibility to attend training was variable. 54\% reported 'easy'/'very easy', 38\% 'difficult'/'very difficult' and 8\% left unanswered.

Conclusion The results of the SENCOs previous training and knowledge of prematurity survey are similar to national research and demonstrated a local training need. ${ }^{1}$

The teaching package was positively received and produced a perceived improvement in knowledge with intended change to future practice to the benefit of local children born prematurely. Study limitations of small numbers and subjective assessment acknowledged and considered for future work.

\section{REFERENCES}

1. Johnson, et al. The long-term consequences of preterm birth: what do teachers know? Developmental Medicine and Child Neurology 2015; 57:571-577

\section{G270 DQ TYPING FOR DOWN SYNDROME SHOULD BE PART OF ROUTINE SURVEILLANCE}

1J Yates, ${ }^{2} P$ Gillett. 'Community Paediatrics, St. John's Hospital, Livingston, UK; ${ }^{2}$ Paediatric Gastroenterology, Royal Hospital of Sick Children, Edinburgh, Edinburgh, UK

10.1136/archdischild-2020-rcpch.234 